\title{
Vaginal Progesterone Gel Versus İntramuscular Progesterone For Luteal Support In Suboptimal Responders According To The Poseidon Criteria (Group 1b), Undergoing Antagonist IVF/ICSI-Cycles.
}

Hasan Ulubasoglu ( $\square$ h.ulubas@hotmail.com )

University of health sciences https://orcid.org/0000-0001-9157-0612

Kadir Bakay

Ondokuz Mayis University

Ali Yavuzcan

Medical Faculty, Düzce University

Davut Guven

Ondokuz Mayis University

\section{Safak Hatırnaz}

Medicana Private Hospital

Michael Dahan

McGill University Health Center

\section{Research Article}

Keywords: luteal phase support,suboptimal responder, POSEIDOIN group I

Posted Date: February 23rd, 2022

DOI: https://doi.org/10.21203/rs.3.rs-1380129/v1

License: (c) (i) This work is licensed under a Creative Commons Attribution 4.0 International License.

Read Full License 


\section{Abstract}

Purpose:The aim of this study is to investigate the efficacy of intravaginal micronized progesterone gel $8 \%$ (VP8) versus intramuscular progesterone in oil (IM P4) for luteal phase support after IVF/ICSI -embryo transfer cycles in POSEIDON suboptimal responder patients.

Methods:We evaluated the data of 189 suboptimal responders who underwent antagonist IVF/ICSI treatments. This retrospective study was conducted on patients who were treated by a university IVF center between January 2010 and December 2015. We evaluated the data of 189 suboptimal responders who underwent antagonist IVF/ICSI treatments. The cycles were categorized into two groups based on the type of luteal support: P4 vaginal gel and P4 IM. Clinical pregnancy, ongoing pregnancy, live birth and spontaneous abortion rates were analyzed.

Results: Patient and treatment characteristics were similar in both groups. However, In terms of infertile duration, significant differences were observed between groups $(P<0.05)$. There were no significant differences in stimulation characteristics including; total gonadotropin doses, peak serum estradiol levels on the day of triggering, number of oocytes collected and number of fertilized oocyte. Multivariate logistic regression modeling failed to find the type of progesterone used as a predictor of live birth (OR 0.802 , $95 \% \mathrm{Cl} 661-1.202, \mathrm{p}=0.580$ ).

Conclusion:We believed in POSEIDOIN group IB patients similar pregnancy outcomes can be obtained by administering $180 \mathrm{mg} \mathrm{P4}$ gel vaginally when compared to IM P4.

\section{Introduction:}

Progesterone (P4) supplementation is required for luteal support in in-vitro fertilization (IVF) cycles, without which pregnancy rates are reduced [1].In natural cycles, P4 is produced by the corpus luteum and is essential for the continuation of the pregnancy. Morover, P4 is responsible for initiating the physiologic endometrial transformations which are mandatory for embryo implantation. P4 protects the embryo by facilitating the production of anti-inflammatory cytokines [2]. If the level of serum P4 is insufficient during implantation, either the embryo can not attach to the endometrium, which would be out of phase or a miscarriage could occur [3].Luteal phase deficiency occurs after IVF procedures, with cycles in the 1970s and 1980s demonstrating low implantation and pregnancy rates until progesterone supplementation was initiated. This occurs for two reasons, injury of the follicles at the time of oocyte retrieval and suppression of endogenous progesterone production. This suppression of progesterone due to the supra physiologic FSH used in stimulation, which results in inhibition of endogenous luteinizing hormone production [4].

There are several routes of administration for P4 supplementation in IVF cycles including vaginal, intramuscular and oral [5]. Higher bioavailability occurs with vaginal and intramuscular administrations than with oral delivery, which is affected by the first pass hepatic metabolism of the P4. The vaginal route is effective in fresh stimulated IVF cycles. In frozen IVF cycles it is debated if vaginal supplementation is as effective as intra muscular injections of P4 [6]. 
Two recent prospective randomized trials suggested that daily intramuscular progesterone was superior to vaginal administration when no corpus luteum was present, due to estrodiol suppression of folliculogenesis in frozen IVF cycles [6,8].

In one of these studies, days of progesterone pre transfer based on type of progesterone differed which may have affected the results. However, when vaginal combined with every third day intra muscular P4 was compared to vaginal P4 only for the same duration, pre treatment outcomes were better when injectable P4 was included [6]. The second study used equivalent duration of progesterone supplementation with the intra muscular arms have higher live birth rates than did the vaginal P4 alone group. As such these result suggested when a corpus luteum was not present intra-muscular injections of progesterone may result in better IVF outcomes as a component of a frozen cycle.

Categorization of patients as normo-responders is often based on the exception of a poor or a high response to ovarian stimulation [9]. Although, a considerable proportion of women with normal ovarin reserve parameters may not manage to reach the optimal range of 10 to 15 oocytes stimulated [8]. Women with 4-9 oocytes are currently viewed as a different outcome group based on the POSEIDON criteria of suboptimal responders [7]. They vary substantially regarding their prognosis compared with women who collected 10 oocytes or more [10,11]. Women in this group demonstrate a live birth rates ranging from $15 \%$ to $36 \%$ depending on the woman's age for the first embryo transfer [11]. However, cumulative, live birth rates are lowers compared with women of the same age with 10-15 oocytes retrieved [11].

The effect of P4 supplementation in the luteal phase of IVF cycles is well established [12]. However, for suboptimal responders is not well studied. In theory women with less follicles stimulated and lower number of corpora luteum may respond closer to frozen cycles without folliculogenesis and may benefit from intra-muscular P4 injections. While there are studies of the administration route of luteal phase support (LPS) in normo responders and Rotterdam criteria poor responders however, there are no studies of the administration route of LPS in suboptimal responders according to the Poseidon criteria.

The aim of this study is to investigate pregnacy outcomes of vaginal crinone $8 \%$ versus IM P4 for luteal phase support in fresh IVF/ICSI cycles in POSEIDON suboptimal responder patients.

\section{Material And Methods:}

\section{Patient Selection}

This study was approved by the ethics committee at the Ondokuz Mayıs University Medical Faculty in Samsun (Approval No:2017/121). This retrospective study included patients who underwent IVF and fresh embryo transfer between January 2010 and December 2015 and who collected 4-9 oocytes. (Poseidon groups 1B). Given its retrospective nature, patient consent was not required. 
Suboptimal responders 23-35 years of age with primary infertility and a body mass index (BMI) of 18$28 \mathrm{~kg} / \mathrm{m} 2$ were included in the study. All women had 4-9 oocytes retrieved after antagonist stimulation with normal ovarian reserve parameters including Anti-Mullerian Hormone greater than $1 \mathrm{ng} / \mathrm{ml}$, or antral follicle counts greater than 10 and basal serum FSH levels less than $10 \mathrm{IU} / \mathrm{L}$ and measured within 6 months of starting stimulation.

Figure 1 demonstrates the inclusion and exclusion criteria. A total of 189 cycles were included in the study. Demographic characteristics, stimulation characteristics and outcomes and pregnancy status were obtained from the patient files. All subjects who received treatment during this time period and fit the inclusion criteria were included in the analysis.

\section{Treatment Protocol:}

Treatment Protocol:

FSH injections were initiated on cycle day 3 if functional ovarian cysts were not present. The initial gonadotropin dose was individualized according to ovarian reserve determined by the antral follicle count, the serum basal FSH level, the female patient's age and the body weight. Ovarian response was observed by serial transvaginal ultrasonogrophy with or without hormonal monitoring. Dosage adjustments were based on the ovarian response. When the leading follicle was $18 \mathrm{~mm}$ and half of follicles of at least $10 \mathrm{~mm}$, were at least $14 \mathrm{~mm}$ in mean diameter, human chorionic gonadotrophin (HCG; Pregnyl. Organon company, the Netherlands) $10000 \mathrm{IU}$ was given intramuscularly to trigger final oocyte maturation. Oocyte collection under transvaginal ultrasound guidance was performed 36 hours later. Fluid was collected from the follicles during oocyte retrieval using a 17-gauge needle and $100 \mathrm{mmHg}$ aspiration pressure. A single embryo with the best morphological grade was transferred into the uterine cavity under ultrasound guidance (GE Logiq Alpha 200). Transferred embryos were day 5, good quality blastocysts, which was characterized by early cavitation, resulting in the formation of an eccentric and expanded cavity lined by a distinct inner-cell mass and trophectoderm layer with a thin zona pellucida. The transfer was performed using a Frydman ultra-soft catheter (CCD Laboratory, France)

\section{Luteal Support:}

Luteal support commenced on the night of oocyte retrieval and continued until the day of pregnancy test. If the test was positive. P4 treatment was continued for up 10 weeks of gestation. The study population was divided into two groups: 76 cycles received micronized natural P4 vaginal gel $180 \mathrm{mg}$ daily (Merck $K G a A)$ (Group 1) and 113 cycles received P4 in oil 100 daily (2ml) IM (injected into the gluteal muscle) (Kocak Farma laboratory, Turkey) (Group 2), for luteal support.

Clinical pregnancy, live birth, ongoing pregnancy rates and spontaneous abortions were analyzed. For the purposes of our study positive $\beta-H C G$ level was defined as $\beta-H C G$ level of at least $20 \mathrm{mlU} / \mathrm{mL}$. The groups were compared with regard to their demographic and stimulation characteristics as well as treatment outcomes. Clinical pregnancy was determined by ultrasound demonstration of cardiac activity at 7 weeks 
gestational age. Spontaneous abortion was defined as the loss of a clinical pregnancy before 12 weeks gestation. An ongoing pregnancy was defined by the identification of fetal heart movements at 12 weeks gestation or after. We defined a live birth as an infant born alive after 24 weeks of gestation who survived more than 28 days.

\section{Statistical analysis:}

Statistical analyzes were performed using SPSS version 25.0 (IBM corp, Chicago, IL). Descriptive statistics were given as median (min: max) frequency and percentage. Data was compared using Chisquare test (for nominal or ordinal scaled data), Fisher exact test (when chi-square test assumptions did not hold due to low expected cell counts). Parametric tests were used for normally distributed data. While nonparametric tests were used for not normally distributed data. Mann - Whitney u tests were used where appropriate. Otherwise Student's T -tests were used to test whether there was a statistically significant difference between numerical variables of the two groups. For the multivariate analyzes, the possible factors identified with univariate analyses were further entered in to the logistic regression analysis to determine independent predictors of live birth rate. Hosmer-Lemeshow tests were used to assess model fit. A $5 \%$ type - I error level was used to interpret as statistically significant.

\section{Results:}

Demographic characteristics of the groups are presented in Table 1 and stimulation data in Table 2. Patient and treatment characteristics were similar in both groups. Mostly, no significant differences were found between the two groups. However, in terms of infertile duration, differences were observed between groups $(P<0.05)$. There were no significant differences in stimulation characteristics such as: mean total gonadotropin doses, serum E2 levels on HCG day, total number of oocytes collected, and the number of fertilized oocyte, between the two groups. Pregnancy outcomes (Table 2) did not differ statistically between the two groups: regarding clinical pregnancy rate per cycle start $(73.7 \%$ and $69.9 .3 \% ; p=0.573)$. Ongoing pregnancy per cycle start or per transfer $(59.2 .1 \%$ and $62.2 \% ; p=0.894)$ and live birth rate per cycle start $(58.1 \%$ and $59.8 \% ; p=0.816)$, respectively. The spontaneous abortion rates $(17.3 \%$ and $15.3 \% ; p=0.714)$ were also similar (Table 2$)$. The effects of different parameters were evaluated on the development of a live birth (table 3). We found that none of the parameters evaluated predicted a live birth including the type of progesterone used (OR 0.802, 95\% $\mathrm{Cl} 661-1.202, \mathrm{p}=0.580$ ).

\section{Discussion:}

In this study, we included patients who underwent fresh single embryo transfer in POSEIDON suboptimal responder women undergoing antagonist IVF/ICSI cycles between the ages of 25-35 years. The pregnancy outcomes for both forms of luteal support were comparable.

In one randomized trial, patients were administered either $\mathrm{P}_{4} 50 \mathrm{mg} \mathrm{IM}$ per day or intravaginal $\mathrm{P}_{4}$ gel per day in normo-responder patients' cycles. The clinical pregnancy rates were comparable however, there 
was a significantly higher incidence of side effects with the intramuscular route of luteal support [13]. Regretfully, data was unavailable from our chart review to determine rates of complications.

Evidence with regard to ideal dose for luteal phase progesterone supplementation is lacking in the literature. Few randomized trials exist to answer this question. Two studies have compared different doses of the vaginal $\mathrm{P}_{4}$ supplementation. Check et al. demonstrated that no differences in clinical pregnancy, ongoing pregnancy, and miscarriage rates were noted while comparing $100 \mathrm{mg}$ and $25 \mathrm{mg}$ of daily IM P4 in a total of 127 patients who were treated with the long GnRH agonist IVF protocol and a fresh transfer [14]. Chanson et al. compared $400 \mathrm{mg}$ versus $600 \mathrm{mg}$ of micronized vaginal $\mathrm{P}_{4}$ and no differences were observed in clinical pregnancy rates in 40 patients who underwent the long $\mathrm{GnRH}$ agonist IVF protocol and a fresh transfer [15].

Several studies have compared outcomes with fresh transfers in IVF based on progesterone supplementation. In one randomized controlled trial, P gel $8 \%(90 \mathrm{mg})$ was compared with $50 \mathrm{mg}$ IM $\mathrm{P}_{4}$ in a normo-responder patient group. Among 194 women studied, those who received $I M \mathrm{P}_{4}$ had higher ongoing pregnancy and live birth rates per transfer, in long protocol fresh IVf cycles [16]. In our study, we compared $\mathrm{P}_{4}$ gel $8 \%\left(180 \mathrm{mg}\right.$ ) versus $100 \mathrm{mg} \mathrm{IM} \mathrm{P}_{4}$ and there was no significant difference in live birth rates or early pregnancy loss rates. Therefore, we believed in POSEIDOIN group IB patients similar pregnancy outcomes can be obtained by administering $180 \mathrm{mg} \mathrm{P}_{4}$ gel vaginally when compared to IM $P_{4}$. We believe in this case the vaginal P4 gel would be preferred due to its favorable side effects profile.

In another study, pregnancy rates and clinical pregnancy rates per transfer were similar for vaginal progesterone gel and IM progesterone groups in Rotterdam poor responders. Women who received vaginal P4 gel had higher rates of biochemical pregnancy loss but lower rates of clinical pregnancy loss than women who received IM progesterone. Whereas, those receiving IM P4 had a higher rate of spontaneous abortion. Ultimately, both forms were associated with similar ongoing pregnancy rates [17]. In our study, there was no significant difference between the two forms in terms of miscarriage rates. The different doses used in our and their studies may explain the different results or more likely this finding was due to bias which occurred due to the small study size in the cited article[19].

Other studies have also failed to find a difference in pregnancy outcomes between the two administration routes $[18,19]$. A recent randomized controlled trial comparing the efficacy of vaginal $\mathrm{P}_{4}$ gel and vaginal micronized P4 tablets compared the pregnancy, clinical pregnancy and take-home baby rates, which were similar in fresh cycles. That study also found that vaginal P4 gel was considered more convenient by the patients [20].

However, studies comparing different vaginal $\mathrm{P}_{4}$ forms with regard to IVF success did not go beyond a limited number [21]. Comprehensive analysis of the present literature revealed a very limited number of studies investigating the differences of the vaginal forms. None had sufficient power to distinguish any difference between the variable forms and doses. 
Two studies provided evidence that there were no differences between the vaginal ring, vaginal gel or tablet groups regarding the clinical pregnancy rates, ongoing pregnancy rate and live birth rates, in women undergoing IVF with normo-responder fresh embryo transfer cycle $[21,22]$.

The small sample size and the retrospective design are the main limitations of our study.

\section{Conclusion:}

According to our knowledge, our study is the first study in the literature to investigate pregnancy outcomes in Poseidon Group 1B patients based on vaginal or IM progesterone supplementation. We suggest that there are similar effects of both P4 forms on the IVF results in suboptimal responder women undergoing antagonist IVF/ICSI cycles. IM P4 supplementation during the luteal phase and earlypregnancy requires prolonged daily injections of $\mathrm{P} 4$, which can lead to patient discomfort, inflammatory reactions and abscesses. As such P4 gel is clearly desirable as an efficient alternative to IM P4. There is a need for randomized control trials and larger studies evaluating different routes of P4 administration and different dosages in poor responder IVF cycles and to confirm our results.

\section{Declarations:}

Hasan Ulubasoglu: project conception, design and and writing of the manuscript.Kadir Bakay:study development Ali Yavuzcan: analysis of the data. Davut Guven: interpretation.Safak Hatırnaz:revising the manuscript.Micheal Dahan :revising it critically for important intellectual content and final approval of the version to be published.

\section{Ethical approval}

All procedures performed in studies involving human participants were in accordance with the ethical standards of the institutional research committee (Approval No:2017/121).

\section{Funding}

No

\section{Disclosure statement}

The authors declare that there are no conflicts of interest.

\section{Conflict of interest statement}

The authors have no conflicts of interest.

\section{References:}


1.Kim CH, Lee YJ, Lee KH (2014) The effect of luteal phase progesterone supplementation on natural frozen-thawed embryo transfer cycles. Obstet Gynecol Sci;57:291-6

2.Szekeres-Bartho J, Wilczynski JR, Basta P ( 2008) Role of progesterone and progestin therapy in threatened abortion and preterm labour. Front Biosci 13:1981-90

3.Bjuresten K. Landgren BM. Hovatta O (2011) Luteal phase progesterone increases live birth rate after frozen embryo transfer. Fertil Steril 95:534-7

4.Daya S (2009) Luteal support: progestogens for pregnancy protection. Maturitas 65:29-34.

5.Beltsos AN, Sanchez MD, Doody KJ (2014) Patients' administration preferences: progesterone vaginal insert (Endometrin $\left.{ }^{\circledR}\right)$ compared to intramuscular progesterone for luteal phase support. Reprod Health $11: 78$

6 .Devine K, Richter KS, Jahandideh S (2021) Intramuscular progesterone optimizes live birth from programmed frozen embryotransfer: a randomized clinical trial. Fertil Steril 12:S0015-0282(21)00298-3

7.Alviggi C, Andersen CY, Buehler K ( 2016) A new more detailed stratification of low responders to ovarian stimulation: from a poor ovarian response to a low prognosis concept. Poseidon Group (PatientOriented Strategies Encompassing Individualized Oocyte Number). Fertil Steril 105(6):1452-3

8. Devine K, Richter KS, Widra EA (2018) Vitrified blastocyst transfer cycles with the use of only vaginal progesterone replacement with Endometrin have inferior ongoing pregnancy rates: results from the planned interim analysis of a three-arm randomized controlled noninferiority trial. Fertil Steril 109:266275

9.Sunkara SK, Rittenberg V, Raine-Fenning N(2011) Association between the number of eggs and live birth in IVF treatment: an analysis of 400135 treatment cycles. Hum Reprod 26:1768-1774

10.Alvaro Mercadal B,Rodríguez I,Arroyo G (2018) Characterization of a suboptimal IVF population and clinical outcome after two IVF cycles. Gynecol Endocrinol 34:125-128

11.Polyzos N.P Sunkara S.K (2015) Sub-optimal responders following controlled ovarian stimulation:an overlooked group. Human Reproduction 30:2005-8

12.Kaser DJ, Ginsburg ES, Missmer SA(2014)Intramuscular progesterone versus 8\% Crinone vaginal gel for luteal phase support for day 3 cryopreserved embryo transfer. Fertil Steril 98:1464-9

13.Anserini P, Costa M, Remorgida V(2001)Luteal phase support in assisted reproductive cycles using either vaginal (Crinone 8) or intramuscular (Prontogest) progesterone: results of a prospective randomized study;Minerva Ginecol 53:297-301 
14.Check JH, Nowroozi K, Chase J(1991) Comparison of luteal- phase support with high- and low-dose progesterone therapy on pregnancy rates in an IVF program. J In Vitro Fert Embryo Transf 8:173-5

15.Chanson A, Germond M, Lagnaux Y(1996) Comparison of two progesterone dose regimens for luteal phase support after embryo transfer: a prospective randomized study. Hum Reprod 11:170

16.Abate A, Perino M,Abate FG (1999) Intramuscular versus vaginal administration of progesterone for luteal phase support after IVF and embryo transfer. A comparative randomized study. Clin Exp Obstet Gynecol 26:203-6

17.Chantilis SJ, Zeitoun KM, Patel SI (1999) Use of crinone vaginal progesterone gel for luteal support in invitro fertilization cycles. Fertil Steril $1999 ; 72: 823-9$

18.Yanushpolsky E, Hurwitz S, Greenberg L(2011) Patterns of luteal phase bleeding in IVF cycles supplemented with Crinone vaginal gel and with intramuscular progesterone impact of luteal estrogen: prospective. randomized study and post hoc analysis. Fertil Steril ;95: 617-620

19.Zarutskie PW. Phillips JA(2009) A meta-analysis of the route of administration of luteal phase support in assisted reproductive technology: vaginal versus IM progesterone. Fertil Steril 92:163-169.

20.Christina Bergh,Svend Lindenberg (2012) Prospective randomized multicenter study comparing vaginal progesterone gel and vaginal micronized progesterone tablets for luteal support after in vitro fertilization/intracytoplasmic sperm injection. Hum Reprod 27:3467-73

21.Bergh C, Lindenberg S, Nordic Crinone Study Group (2012) A prospective randomized multicentre study comparing vaginal progesterone gel and vaginal micronized progesterone tablets.Hum Reprod 27:3467-73

22.Stadtmauer L, Silverberg KM,Ginsburg ES (2013)Progesterone vaginal ring versus vaginal gel for luteal support with in vitro fertilization: a randomized comparative study. Fertil Steril 99:1543-9

\section{Tables:}

Table 1. Demographic characteristics of the patients in the groups 


\begin{tabular}{|c|c|c|c|}
\hline & $\begin{array}{l}\text { Progesterone gel } \\
(\mathrm{n}: 76)\end{array}$ & $\begin{array}{l}\text { Progesterone ampoule } \\
(\mathrm{n}: 113)\end{array}$ & $\mathrm{p}$ \\
\hline Age (years);(min-max) & $30(23: 35)$ & $23(23: 35)$ & $0,323^{a}$ \\
\hline $\begin{array}{l}\text { Duration of infertility (years);(min- } \\
\text { max) }\end{array}$ & $6(2: 8)$ & $5(1: 8)$ & $0,043^{a}$ \\
\hline $\begin{array}{l}\text { Number of antral follicles on day } 3 ; \\
\text { (min-max) }\end{array}$ & $8(5: 14)$ & $8(5: 12)$ & $0,743^{a}$ \\
\hline D3 FSH [IU/L];(min-max) & $6(4: 10)$ & $6(4: 10)$ & $0,573^{a}$ \\
\hline D3 estradiol [pg/mL];(min-max) & $36,5(5: 74)$ & $36,0(8: 66)$ & $0,593^{a}$ \\
\hline $\operatorname{BMI}\left(\mathrm{kg} / \mathrm{m}^{2}\right) ;(\min -\max )$ & $21(17-27)$ & $17(17-27)$ & $0,060^{\mathrm{a}}$ \\
\hline Infertility diagnosis, n (\%) & & & $0,323^{b}$ \\
\hline Unexplained & $40(\% 52,6)$ & $75(\% 66,4)$ & \\
\hline Male factor & $32(\% 42,1)$ & $34(\% 30,1)$ & \\
\hline Tubal & $4(\% 5,3)$ & $4(\% 3,5)$ & \\
\hline
\end{tabular}

${ }^{*} \mathrm{P}<0,05$ was accepted significant.

Data are presented as median (min: max) and number (percent).

a: Mann-Whitney U test, b: $\chi 2$ test.

hCG - human chorionic gonadotropin ,FSH - follicle stimulating hormone, E2 - estradiol, BMI -Body Mass Index, D-day

Table 2. Stimulation characteristics and treatment outcomes of 198 cycles 


\begin{tabular}{|llll|}
\hline & $\begin{array}{l}\text { Progesterone } \\
\text { gel(n:76) }\end{array}$ & $\begin{array}{l}\text { Progesterone } \\
\text { ampoule(n:113) }\end{array}$ & P \\
\hline Total gonadotropin dose (IU);(min-max) & $\begin{array}{l}3125 \\
(2500: 4250)\end{array}$ & $3200(2250: 4900)$ & $0.412^{\mathrm{a}}$ \\
\hline $\begin{array}{l}\text { Endometrial thickness on the day of HCG } \\
\text { (mm);(min-max) }\end{array}$ & $9(7: 16)$ & $10(7: 14)$ & $0.535^{\mathrm{a}}$ \\
\hline $\begin{array}{l}\text { Total Duration of stimulation (days);(min- } \\
\text { max) }\end{array}$ & $10(8.13)$ & $10(8.14)$ & $0.6^{\mathrm{a}}$ \\
\hline $\begin{array}{l}\text { Serum E2 on the day of HCG [pg/mL]:(min- } \\
\text { max) }\end{array}$ & $\begin{array}{l}2000 \\
(1600: 2100)\end{array}$ & $2000(1750: 2900)$ & $0.444^{\mathrm{a}}$ \\
\hline $\begin{array}{l}\text { Number of oocytes retrieved ;(min-max) } \\
\text { Number of MIl oocytes ;(min-max) }\end{array}$ & $7(4: 9)$ & $7(4: 9)$ & $0.775^{\mathrm{a}}$ \\
\hline $\begin{array}{l}\text { Number of fertilized oocytes }(2 \mathrm{PN}) ;(\min - \\
\text { max) }\end{array}$ & $6(1: 9)$ & $6(2: 9)$ & $0.937^{\mathrm{a}}$ \\
\hline $\begin{array}{l}\text { Clinical pregnancy rate/per transfer ;(min- } \\
\text { max) }\end{array}$ & $(\%) 73.7$ & $5(1: 9)$ & $0.260^{\mathrm{a}}$ \\
\hline Ongoing pregnancy rate/cycle & $(\%) 59.2$ & $(\%) 69.9$ & $0.573^{\mathrm{b}}$ \\
\hline Live birth rate/cycle & $(\%) 58.1$ & $(\%) 62.2$ & $0.894^{\mathrm{b}}$ \\
\hline Spontaneous abortion per pregnancy & $(\%) 17.3$ & $(\%) 15.3$ & $0.816^{\mathrm{b}}$ \\
\hline
\end{tabular}

Data are presented as median (min: max) and number (percent).

a; Mann-Whitney U test. b; $\chi 2$ test.

hCG - human chorionic gonadotropin. MII-Metaphase II stage, 2PN: 2 pro nuclei on retrieval day 1.

Table:3 Evaluation of Predictors of a live birth** 


\begin{tabular}{|llc|}
\hline Independent variables & OR* 95 \%(Lower-Upper) & $\mathbf{P}$ \\
\hline Type of progesterone used for luteal support & $0.802(661-1.202)$ & .580 \\
\hline Serum Day 3 estradiol & $0.997(978-1.017)$ & .772 \\
\hline Female Age & $0.957(895-1.023)$ & .199 \\
\hline Serum P4 on the day of HCG & $0.999(998-1.001)$ & .367 \\
\hline Total duration of induction & $1.010(0.821-1.243)$ & .923 \\
\hline Serum E2 on the day of HCG & $1.001(1.000-1.001)$ & .110 \\
\hline Number of total oocytes retrieved & $1.196(944-1.515)$ & .137 \\
\hline Number of Mature oocytes (MII) & $1.380(983-1.936)$ & .063 \\
\hline Average gonadotropin dose per day & $1.012(526-1.948)$ & .971 \\
\hline Total Gonadotropin Dose used for stimulation & $1.000(999-1.001)$ & .528 \\
\hline Number of embryo retrieved & $0.902(663-1.226)$ & .510 \\
\hline
\end{tabular}

*OR: Odds ratio and $95 \%$ confidence interval

** Multivariate logistic regression analysis with live birth rate as dependent (outcome) variable.Age, BMI, infertility duration were controlled as confounding factors.

\section{Figures}




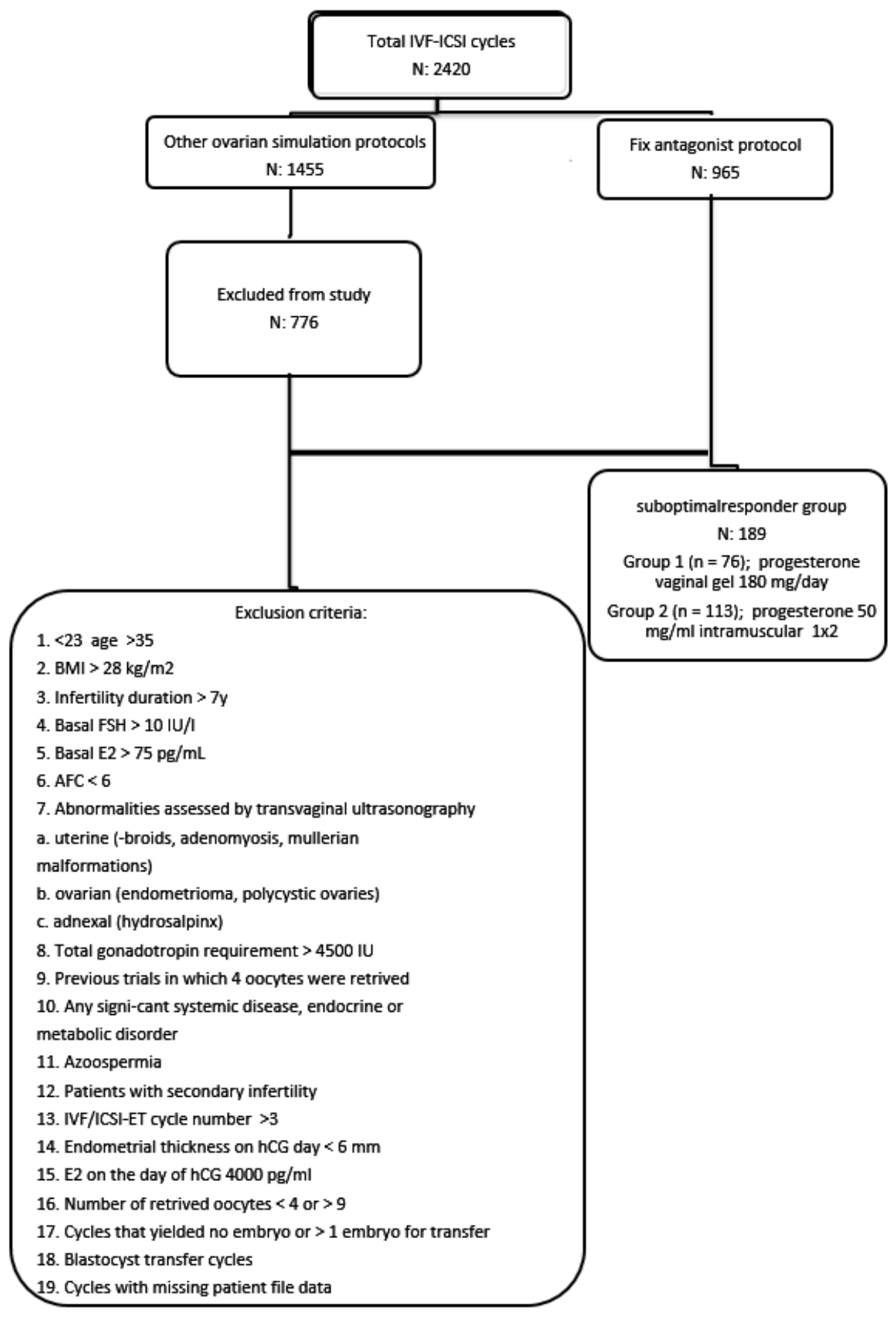

\section{Figure 1}

Flow chart of the study: included and excluded cycles. BMI - body mass index, E2 - estradiol, AFC antral follicle count 